\title{
BOUNDARY PROPERTIES OF MINIMAL HARMONIC FUNCTIONS
}

\author{
JOHN T. KEMPER
}

\begin{abstract}
Certain elements of the boundary dependence of minimal harmonic functions in Euclidean domains are considered. For a given minimal harmonic function $h$ on a domain $\Omega$, sets on the boundary of $\Omega$ or (relative) neighborhoods of such sets are sought wherein the behavior of $h$ determines $h$ in all of $\Omega$. The set of determining singletons on the boundary is shown to be connected.
\end{abstract}

A minimal harmonic function in a Euclidean domain $\Omega$ (introduced by Martin [4]) is a positive harmonic function $h(x)$ in $\Omega$ satisfying the "minimal" property:

if $u(x)$ is any harmonic function in $\Omega$ such that $0 \leqslant u \leqslant h$

in $\Omega$, then there is a constant $C \geqslant 0$ such that $u=C h$.

Martin proved that these functions form a basis for all nonnegative harmonic functions in $\Omega$, with any such function represented by an integral of minimal harmonic functions with respect to a uniquely determined measure. In the case of domains $\Omega$ with smooth boundaries, this representation is the Poisson integral formula and the minimal harmonic functions are the Poisson kernel functions with poles on $\partial \Omega$. This equivalence of the "Martin boundary" and the topological boundary was shown to hold, more generally, in Lipschitz domains by Hunt and Wheeden [3].

Attempts to specify precisely the relationship between minimal harmonic functions and points on $\partial \Omega$ have not been completely successful. Among the more important contributions in this area is a result of Brelot [1]:

(1) THEOREM. If $h$ is a minimal harmonic function in a bounded domain $\Omega$, then there is at least one point $y \in \partial \Omega$ such that $h$ is associated to $y$ in the sense that $R_{h}^{y}=h .\left(R_{h}^{y}\right.$ is the "reduced function for $h$ at $y$ "; see [2] for an account of the properties of reduced functions.)

One of the objects of this report is to obtain information about the set of points $y \in \partial \Omega$ which are associated with a particular minimal harmonic

Received by the editors June 10, 1975 and, in revised form, February 23, 1976.

AMS (MOS) subject classifications (1970). Primary 31B25; Secondary 31B10, $35 \mathrm{~J} 05$.

Key words and phrases. Minimal harmonic functions, Poisson kernel functions. 
function. For the sake of comparison we first recall a more classical association of harmonic functions in $\Omega$ to points of $\partial \Omega$ : a nonnegative harmonic function $u$ in $\Omega$ has a pole at $y \in \partial \Omega$ if $\limsup _{x \rightarrow y ; x \in \Omega} u(x)=\infty$. We can easily verify that the poles of a minimal harmonic function cannot be arbitrarily distributed.

Proposition. If $h$ is a minimal harmonic function in a bounded domain $\Omega$, then the set of poles of $h$ is a connected subset of $\partial \Omega$.

Proof. The set $P=\{y \in \partial \Omega: y$ is a pole of $h\}$ is a closed subset of $\partial \Omega$. If $y_{0} \in \partial \Omega$ is one point (guaranteed by (1)) which is associated to $h$, it is clear from the construction of the reduced function that $y_{0} \in P$. Let $Y$ be the component of $P$ containing $y_{0}$. Choose an open set $\Gamma$ such that

$1^{\circ} . Y$ is contained in $\Gamma$,

$2^{\circ} . P \backslash Y$ is contained in $(\tilde{\Gamma})^{0}$, the interior of $\tilde{\Gamma}$,

$3^{\circ} . \partial \Gamma$ is smooth.

Since $y_{0} \in \Gamma$, we have $R_{h}^{\Omega \cap \bar{\Gamma}}=h$, and it follows that $h$ is equal in $\Omega \backslash \bar{\Gamma}$ to the solution of the Dirichlet problem in that set with boundary values

$$
\varphi(x)= \begin{cases}h(x) & \text { for } x \in \partial \Gamma \cap \Omega, \\ 0 & \text { for } x \in \partial \Omega \backslash \Gamma\end{cases}
$$

Since $\varphi(x)$ is bounded, the maximum principle assures that $h$ is bounded in $\Omega \backslash \bar{\Gamma}$ and, therefore, $P=Y$ is connected.

In order to consider the connectivity question for the set of boundary points associated to a minimal harmonic function (in the sense of (1)) we first develop a useful consequence of the "minimal" nature of these functions.

(2) Lemma. Let $\Omega$ be a bounded domain in $\mathbf{R}^{n}$ and $\Gamma$ a closed subset of $\bar{\Omega}$. If $h$ is a minimal harmonic function in $\Omega$ with $R_{h}^{\Gamma \cap \Omega}=h$, then there is a point $\xi \in \Gamma \cap \partial \Omega$ such that $R_{h}^{\xi}=h$. (For example, if a "slice" of $\Omega$ contains enough information to generate $h$, there must be a point of $\partial \Omega$ in that "slice" which is associated to h.)

Proof. Let $W$ be an arbitrary neighborhood (in $\Omega$ ) of $\Gamma \cap \partial \Omega$ and suppose that $u(x) \geqslant 0$ is a superharmonic function in $\Omega$ such that $u \geqslant h$ in $W$. If $M=\sup \{h(x): x \in(\Gamma \cap \Omega) \backslash W\}$, which is finite, then $v(x)=u(x)+2 M$ is a positive superharmonic function in $\Omega$ with $v(x) \geqslant h(x)$ in a neighborhood of $\Gamma$ in $\Omega$. Since $R_{h}^{\Gamma \cap \Omega}=h, v(x) \geqslant h(x)$ for all $x \in \Omega$. Because $h$ is minimal, there is a $y \in \partial \Omega$ satisfying $R_{h}^{y}=h$, and we have

$$
h=R_{h}^{y} \leqslant R_{v}^{y} \leqslant R_{u}^{y}+R_{2 M}^{y}=R_{u}^{y} \leqslant u \text { in } \Omega .
$$

It follows that $h \leqslant R_{h}^{W}=\inf \{u \geqslant 0$, superharmonic in $\Omega: u \geqslant h$ on $W\}$ and, therefore, $h=R_{h}^{\Gamma \cap \partial \Omega}$.

Because $h$ is minimal, for any set $A \subset \partial \Omega$, either $R_{h}^{A}=h$ or $R_{h}^{A}=0$. If $\xi \in \Gamma \cap \partial \Omega$ and $R_{h}^{\xi}=0$, there must be a set $\theta_{\xi}$, open in $\partial \Omega$, with $\xi$ $\in \mathcal{O}_{\xi}$ and $R_{h}^{\mathfrak{Q}_{\xi}}<h$, hence $R_{h}^{\mathfrak{O}_{\xi}}=0$. Suppose that $R_{h}^{\xi}=0$ for every $\xi \in \Gamma$ 
$\cap \partial \Omega$. Since $\Gamma \cap \partial \Omega$ is compact, there exist $\xi_{1}, \xi_{2}, \ldots, \xi_{k} \in \Gamma \cap \partial \Omega$ such that $\Gamma \cap \partial \Omega \subset \cup_{i=1}^{k} \vartheta_{\xi_{i}}$ and, therefore,

$$
R_{h}^{\Gamma \cap \partial \Omega} \leqslant R_{h}^{\cup \cup_{i=1}^{k} \theta_{\xi_{i}}} \leqslant \sum_{i=1}^{k} R_{h}^{\vartheta_{\xi_{i}}}=0
$$

a contradiction. Hence there is at least one $\xi \in \Gamma \cap \partial \Omega$ with $R_{h}^{\xi}=h$.

We can now prove our main result.

(3) THEOREM. If $h$ is a minimal harmonic function on $\Omega$, a bounded domain in $\mathbf{R}^{n}$, then the set $E=\{y \in \partial \Omega: y$ is associated to $h\}$ is connected.

Proof. Suppose that $E=E_{1} \cup E_{2}$, where $E_{1}, E_{2}$ are closed, nonempty, disjoint subsets of $\partial \Omega$. Let $\Gamma_{1} \subset \Gamma_{2}$ be compact neighborhoods of $E_{1}$ in $\bar{\Omega}$ with $\Gamma_{1} \subset \Gamma_{2}^{0}$ and $\Gamma_{2} \cap E_{2}=\varnothing$. Set $\Gamma=\Gamma_{2} \backslash \Gamma_{1}^{0}$. Since $E_{1}$ and $E_{2}$ are nonempty sets of boundary points associated to $h$, we have

$$
R_{h}^{\Gamma_{h} \cap \Omega}=R_{h}^{\Omega \backslash \Gamma_{2}^{0}}=h .
$$

Let $u \geqslant 0$ be a superharmonic function in $\Omega$ with $u \geqslant h$ on $\Gamma \cap \Omega$. Then

$$
w(x)= \begin{cases}h(x) & \text { for } x \in \Gamma_{2}, \\ \min (h(x), u(x)) & \text { for } x \in \Omega \backslash \Gamma_{2},\end{cases}
$$

is a nonnegative superharmonic function in $\Omega$ with $w \geqslant h$ on $\Gamma_{2}$. It follows that

$$
u(x) \geqslant W(x) \geqslant R_{h}^{\Gamma_{2}}(x)=h(x) \text { for } x \in \Omega \backslash \Gamma_{2} .
$$

Similarly, $u(x) \geqslant h(x)$ for $x \in \Gamma_{1}^{0} \cap \Omega$, and, therefore, $R_{h}^{\Gamma \cap \Omega}=h$. By Lemma (2), there must be a point $\eta \in \Gamma \cap \partial \Omega$ which is associated to $h$; i.e., $\eta \in E$. This is impossible since $E=E_{1} \cup E_{2}$ has empty intersection with $\Gamma$, and we conclude that $E$ must be connected.

In the case of an unbounded domain $\Omega$, a few modifications are necessary. Brelot's Theorem (1) in that case allows for a minimal harmonic function to be associated to $\infty$, the additional boundary point obtained in the one point compactification of $\bar{\Omega}$. For a particular minimal harmonic function $h$ in $\Omega$, this means

$$
\begin{aligned}
& R_{h}^{\Omega_{r}}=h \text { in } \Omega \text { for every value of } r \text { sufficiently large, } \\
& \text { where } \Omega_{r}=\Omega \cap\left\{x \in \mathbf{R}^{n}:|x| \geqslant r\right\} .
\end{aligned}
$$

For example, the function $h(x, y)=y$ in the upper half-plane is minimal and is not associated to any finite boundary point, but does satisfy (4).

The analog of Theorem (3) for unbounded domains can now be stated.

THEOREM. If $h$ is a minimal harmonic function in an unbounded domain $\Omega \subset \mathbf{R}^{n}$, then the set $E \subset \partial \Omega \cup\{\infty\}$ of points to which $h$ is associated is 
connected. (The topology on $\partial \Omega \cup\{\infty\}$ is the one induced by the compactification.)

Proof. If $E$ a bounded set, the arguments in (2) and (3) are essentially unchanged. If $E$ is unbounded and disconnected, let $E=E_{1} \cup E_{2}$ as in the proof of (3), with $E_{2}$ the unbounded component. Choose $\Gamma_{1}$ and $\Gamma_{2}$ to be compact, bounded neighborhoods of $E_{1}$ with $\Gamma_{1} \subset \Gamma_{2}^{0}$ and $\Gamma_{2} \cap E_{2}=\varnothing$. Then $\Gamma=\Gamma_{1} \backslash \Gamma_{2}^{0}$ is bounded with $R_{h}^{\Gamma \cap \Omega}=h$. Lemma (2) follows as before as long as care is taken to use a point of $E_{1}$ for the point $y$ associated to $h$, in which case $R_{2 M}^{y}$ is again zero. The lemma and theorem are then completed as before.

In case $n=2$, a minimal harmonic function in a domain which is conformally equivalent to the unit disk has exactly one associated boundary point, as minimal harmonic functions are preserved under conformal mapping and the situation in the disk is well known.

\section{REFERENCES}

1. M. Brelot, Le problème de Dirichlet axiomatique et frontière de Martin, J. Math. Pures Appl. (9) 35 (1956), 297-335. MR 20 \#6607.

2. - On topologies and boundaries in potential theory, Lecture Notes in Math., vol. 175, Springer-Verlag, Berlin and New York, 1971. MR 43 \# 7654.

3. R. A. Hunt and R. L. Wheedon, Positive harmonic functions on Lipschitz domains, Trans. Amer. Math. Soc. 147 (1970), 507-527. MR 43 \#547.

4. R. S. Martin, Minimal positive harmonic functions, Trans. Amer. Math. Soc. 49 (1941), 137-172. MR 2, 292.

School of Mathematics, University of Minnesota, Minneapolis, Minnesota 55455 